\title{
Fault Diagnosis of Aircraft Power Starting System Based on MTBF-SVM
}

\author{
Liang Qin ${ }^{*}$, Zhen Wang and Xian-Jun Shi
}

Department of Control Engineering, Naval Aeronautical and Astronautical University, Yantai 264001, China

\author{
Keywords: SVM, Starting System, Fault Diagnosis
}

\begin{abstract}
In order to solve the fault diagnosis of aircraft power starting system, the fault diagnosis method through constructing binary tree SVM (support vector machines) is researched in this paper. Consider that the components which have high fault rate have priority to be isolated, the method trains the classifier depend on MTBF from small to big and uses the basic structure of binary tree-SVM to generate the leaf nodes gradually, each leaf node represents a fault mode. The method is applied to the identify the common nine fault mode of aircraft power starting system.
\end{abstract}

\section{Introduction}

The power starting system is the important airborne equipment of aircraft and the key system which starts the aircraft engine timely and reliably in the ground and air[1]. Therefore, locate the fault of aircraft power starting system fast, accurately and reliably is of importance to guarantee the safety of aircraft flight and improve the combat effectiveness of the army.

Literature [2] establishes the Petri net model of aircraft power starting system to reasoning the fault expert system, the results prove that it improve the diagnosis efficiency. Through fusing the data of different sensors, Literature [3] uses the DS evidence theory to research the fault diagnosis problems of aircraft power system. The above methods use many prior knowledge, they usually don't take the promotion of the method into consideration.

Because the support vector machine(SVM)[4] which has been widely applied to the fault diagnosis field in recent years follows the basic principle of structure risk minimization and has strong generalization ability, it can solve many practical problems such as small sample problems, non-linear problems, high dimension problems, local minimum problems and so on[5]. Apart from this, it overcomes the shortcut of other self-learning methods(neural net method) such as long learning time, over-learning easily and so on. The method provides a new theoretical way and technology realization mean for modern complicated large system. This paper take a research on fault diagnosis of aircraft power starting system based on SVM and the method can improve the fault diagnosis efficiency.

\section{The Basic Principle of MTBF-SVM}

Since 1995 Vapnik proposed SVM it has always been taken attention in the theoretical research and engineering application. The initial SVM algorithm is used to construct an optimal hyper which distinguished two classes of samples. But multi-classification problems occur in practical application, the basic idea of the current common algorithm is that divide a multi-classification problem into two classis classification problem, then classifies the sub-classifier through combination in a certain way. Nowadays one-against-one algorithm[6], one-against-rest algorithm[7] and once for all algorithm is widely used and their performance is well. However, there exist a large number of regions which can't be separate in these algorithms, and their training time and test time is very long. Aim at the problem, this paper puts forward to a binary tree-SVM algorithm based on average fault rate, and applies to fault diagnosis of aircraft power starting system. 


\section{The Principle}

The basic principle of binary tree-SVM is that all samples are divided into two sub-classes at first and forms a leaf node and a branch node, then the branch node is divided into two sub-categories further, goes on cycling, until all the nodes contains only one class of samples, namely the last leaf node, thus forms an inverted binary classification tree. Binary tree-SVM separates multi-classification problems into multiple two classification problem, it need construct k-1 SVM classifier for $\mathrm{k}$ classification problem. This method overcomes impartibility problem which many traditional multi-classification methods encounters, and the decision stage doesn't necessarily need to calculate all the discriminant functions of classifier, thus it can save the test time.

When the binary tree SVM occurred, it was widely used and achieved good results, but with the further research, the structure of binary tree becomes a new research hot-spot. A large number of research show that different binary tree structures have different classification model and the efficiency of decision will have significant difference. Figure 1 is two different binary tree structures which are formed by four-classification problem, assume that the classification samples which have not been classified belong to $\mathrm{n} 1$ classification, it needs only one classifier to calculate the result for the decision tree which is formed by (a), but it needs three for (b). Literature [8] points out that different binary tree structures have different classification models, and the segment regions of each classification is different, their promotion performance is different.

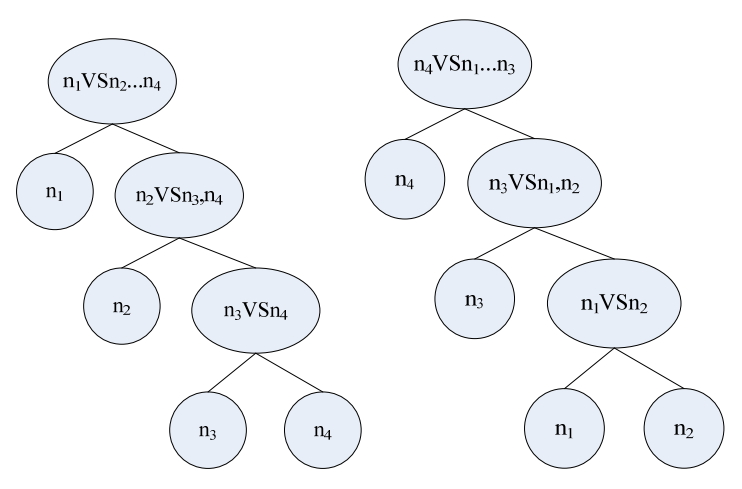

Figure 1. Binary tree classifier structures

This paper uses binary tree SVM to solve the fault diagnosis of aircraft power starting system. Under the condition that there is no fault reasoning, the mode of high fault rate should be separated from all the modes in the fault diagnosis problems, and so forth, separate the mode of the highest fault rate from remaining modes every time, then construct a binary tree based on fault rate. For fault diagnosis problem, the above idea also conforms to the common way of thinking that suspect the low reliable and high fault rate fault mode at first. When The mode has the higher fault rate, it will locate in the upper node of binary tree, this method can improve the efficiency of fault diagnosis.

This paper's method selects the mean time between faults (MTBF) as the basis to construct the binary tree from the parameters which represents the reliability of equipment.

$N_{0}$ fault occurs for a repairable product during use period, after repairing it will continue to work each time, the working hours are respectively $t_{1}, t_{2}, \cdots, t_{N_{0}}$, then its MTBF is

$$
T_{B F}=\frac{1}{N_{0}} \sum_{i=1}^{N_{0}} t_{i}=\frac{T}{N_{0}}
$$

In above formula, $T_{B F}$ is $\operatorname{MTBF}(\mathrm{h}),{ }^{T=\sum_{i=1}^{N_{0}} t_{i}}$ is the total working hours. 


\section{Steps}

According to the above principle, the algorithm process of MTBF-SVM is

Step 1: According to the historical data, calculate the MTBF of the whole components.

Step 2: Select the fault mode ${ }^{S_{i}}$ corresponding to the component which has the smallest MTBF

Step 3: Take ${ }^{S_{i}}$ sample points as positive class and the rest sample points as negative class to train classifier, the ${ }^{S_{i}}$ sample points are removed from the total sample after the completion of training

Step 4: If there are $N-1$ classifiers after training, the training is over, or continue to step 2.

\section{Fault Diagnosis of Aircraft Power Starting System}

In the basis of research and demonstration for aircraft practical work, combined with the self-characteristic of aircraft power starting system, select the common nine kinds of fault modes (the normal system mode is included), as shown in Table 1.

\begin{tabular}{ll}
\multicolumn{2}{c}{ Table 1. the fault reason and its corresponding code } \\
\hline S1 & System Normal \\
\hline S2 & Starting Button fault \\
\hline S3 & "Ground Starting Switch" fault \\
\hline S4 & Timing Mechanism fault \\
\hline S5 & Ignition Coil fault \\
\hline S6 & Relay does not convert \\
\hline S7 & Gasoline Pump or Gasoline Value fault \\
\hline S8 & Exciting Voltage is too low \\
\hline S9 & Contactor does not convert \\
\hline
\end{tabular}

Each fault mode has 50 sample points in the experiment that 30 sample points are regarded as training sample points and the others are regarded as decision sample points, in order to avoid the classification surface shifting which is caused by the number of sample points, generally select 300 sample points to experiment. Using the radial basis kernel function $K(x, y)=\exp \left(-\|x-y\|^{2} / 2 \sigma^{2}\right), e=0.01$, use the combination of different kernel parameter $C$ and $\sigma$, namely select the numerical value crosswise from $C=\left[2^{0}, 2^{1}, \cdots, 2^{5}\right]$ and $\sigma=\left[2^{-1}, 2^{0}, 2^{1}, \cdots, 2^{5}\right]$, the highest diagnostic rate is chosen as experiment data.

Firstly, regard the system normal sample points and the fault sample points as two kinds of samples to train, and form the first classifier. Then according to the MTBF, select the sample points and the rest sample points successively to train and form the classifiers.

Table 2 lists the total sample number of different fault mode and support vector number which is formed by training. The support vector number is apparently less than the total sample number, it illustrates that the diagnosis model has good generalization ability.

Table 2. The train results

\begin{tabular}{lccccc}
\hline Fault & Number of fault & Number of SV & Fault & Number of fault & Number of SV \\
\hline S1 & 600 & 57 & S6 & 150 & 31 \\
S2 & 270 & 41 & S7 & 120 & 28 \\
S3 & 240 & 35 & S8 & 90 & 20 \\
S4 & 210 & 37 & S9 & 60 & 11 \\
S5 & 180 & 35 & S10 & 60 & 11 \\
\hline
\end{tabular}

In the basis of forming the classifiers, apply decision rule to justify the fault mode in the diagnostic process, and conduct experiment for non-training sample. Using the diagnostic rate to represent ability, the diagnostic rate is the proportion which fault sample is classified correctly, the higher the 
diagnostic rate, the stronger the diagnosis ability of the future new sample. The results are shown in Table 3.

Table 3 The diagnosis result

\begin{tabular}{cccccc}
\hline \multirow{2}{*}{ fault } & \multicolumn{2}{l}{ Diagnostic rate } & \multirow{2}{*}{ fault } & \multicolumn{3}{c}{ Diagnostic rate } \\
\cline { 2 - 3 } \cline { 5 - 6 } & Neural net & This paper's method & & Neural net & This paper's method \\
\hline 1 & 0.91 & 0.95 & S6 & 0.75 & 0.86 \\
S2 & 0.84 & 0.91 & S7 & 0.83 & 0.88 \\
S3 & 0.77 & 0.85 & S8 & 0.88 & 0.89 \\
S4 & 0.79 & 0.90 & S9 & 0.87 & 0.91 \\
S5 & 0.74 & 0.88 & S10 & 0.85 & 0.93 \\
\hline
\end{tabular}

In the diagnosis experiment, compared with neural net method, the results show that this paper's method has the higher diagnostic rate for decision sample points which don't take part in training.

\section{Conclusion}

This paper conducts the research on fault diagnosis problems of aircraft power starting system, according to the characteristic object, this paper puts forward to fault diagnosis method based on binary tree SVM which regards $\mathrm{MTBF}$ as index, the method gives full play to the strong generalization merit of SVM, and improves the performance under multi-classification condition. The results show that MTBF-SVM has good effect in the fault diagnosis of aircraft power starting system.

\section{References}

[1] Yang Yinghua, Tang Daquan, Lu Jianhua. The application and developing trend of neural network in intelligent fault diagnosis technology [J]. Journal of measurement and control technology, 2003,22(9):1-5.

[2] Peng Kanghua, Meng Yuxing. The application of fuzzy Petr net model in fault diagnosis of aircraft power starting system[J]. China Power Education, 2007, Research and Technology Forum: 280-282.

[3] Cheng Jianxing, Shi Yikai. Fault diagnosis of aircraft power starting system based on multi-sensor data fusion[J]. Fire and command control, 2012,8(37):184-187.

[4] Vapnik V N. An overview of statistical learning theory[J]. IEEE Trans. On Neural Networks(S1045-9227), 1999, 10(5): 988-999.

[5] Chapelle, V.Vapnik, O Musquet, et al. Choosing Multiple Parameters for Support Vector Machines [J], Machine Learning, 2002(46):131-159.

[6] Vapnic V N. Statistical learning theory[M]. New York:wiley,1998.

[7] Krebel U. Pairwise classification and support vector machines[M]. Cambridge: The MIT Press, 1999: 255-268.

[8] Tang Faming, Wang Zhongdong, Chen Jinyun. A new multi-classification SVM algorithm based on binary tree[J]. Computer engineering and Application, 2005, 7: 24-26. 